\title{
CONCENTRATION OF BINARY FM SPECTRA
}

\author{
P. PAPANTONI-KAZAKOS \\ Electrical Engineering Dept. \\ Rice University \\ Houston, Texas
}

Summary -- The spectrum of a digital FM signal can be considered as an indicator of the resistance of the signal to distortions caused by band-limitation. The study in this paper is oriented toward the design of signaling pulses that will achieve an FM signal spectrum concentrated about the carrier frequency. Convenient spectrum expressions and general guarantees of optimality are found for binary FSK signals.

Introduction -- The frequency shift keying systems have been the subject of many studies the past few years [1-3]. In these systems, the modulation signal is a series of pulses given by

$$
\sum_{i} a_{i} g(t-n T)
$$

where $g(t)$ gives the shape of the arbitrary pulse of duration $T$ and $a_{i}$ are the information digits. The modulated waveform is a sinusoidal process $\mathrm{S}(\mathrm{t})$ given by the following expressions

$$
\begin{aligned}
& S(t)=A \cos \left(\theta+\omega_{c} t+\omega_{d} \sum_{i=1}^{m} a_{i} L+\omega_{d} a_{m+1} \int_{0}^{t-m T} g(\tau) d \tau\right) \\
& \text { for } m T \leq t \leq(m+1) T
\end{aligned}
$$

where A : the constant amplitude;

$\omega_{c}:$ the carrier frequency;

$\omega_{\mathrm{d}}:$ the transmission frequency;

$a_{i}$ : the information digit transmitted during the time interval [(i-1)T,iT];

$\mathrm{L}$ : the pulse energy in $[0, \mathrm{~T}]$, taken in general equal to one;

$\theta$ : the initial phase.

The waveform $S(t)$ goes through two band-pass filters with spectra concentrated about the frequencies $\pm \omega_{c}$ before the process of detecting the digits $a_{i}$ starts. The first band-pass filter is an element of the transmitter and it is necessary for cutting down in wasteful power 
of transmission, while the second band-pass filter is the first receiver element and it is necessary for banding the spectrum of the channel noise.

The less concentrated the spectrum of the waveform $S(t)$ is about the $\pm \omega_{c}$ frequencies, the more $\mathrm{S}(\mathrm{t})$ is distorted due to band limitation, and the larger the probability of false detection in the system [4]. The waveform $S(t)$ being a function of the pulse shape $g(t)$, proper pulse design will result in concentrated "optimized" spectrum.

Convenient expressions of the $\mathrm{S}(\mathrm{t})$ autocorrelation and spectrum will be sought with emphasis on the binary case $\left(a_{i}= \pm 1\right.$ with probability $1 / 2$ each). Effort will be given to establishing pulse characteristics that will "optimize" the above expressions.

In this paper, only the case of the $\mathrm{a}_{\mathrm{i}}$ digits being independent will be covered. In this case the mean $E\{S(t+\tau) S(t)\}$ can easily be found. The waveform $S(t)$ is not a stationary process. To stationarize it, one must average with respect to $\tau$. Before this is done, the mean $\mathrm{E}\left\{\exp \left(\mathrm{j} 2 \omega_{\mathrm{d}} \mathrm{a}_{\mathrm{i}}\right)\right\}$ will be discussed.

The phase interval $[-\pi, \pi]$ is available for transmission, so one has to choose all the possible values of $\omega_{\mathrm{d}} \mathrm{a}_{\mathrm{i}}$ in it. If the middle and edge points of the interval are excluded, one has that

$$
-\pi<\omega_{\mathrm{d}} \mathrm{a}_{\mathrm{i}}<\pi, \omega_{\mathrm{d}} \mathrm{a}_{\mathrm{i}} \neq 0
$$

Let us notice that in $\omega_{\mathrm{d}} \mathrm{a}_{\mathrm{i}}$ the frequency $\omega_{\mathrm{d}}$ is multiplied by the unit time and has phase dimensions. The exclusion of the points $-\pi, 0, \pi$ does not impose any actual restrictions to the problem. Furthermore, if the information digits have density symmetric about the origin, then

and

$$
E\left\{\exp \left(j x a_{i}\right)\right\}=E\left\{\exp \left(-j x a_{i}\right)\right\}
$$

$$
-1 \leq \mathrm{E}\left\{\exp \left(\mathrm{j} 2 \omega_{\mathrm{d}} \mathrm{a}_{\mathrm{i}}\right)\right\}<1
$$

If expression $E\{S(t+\tau) S(t)\}$ is averaged with respect to $t$, and expressions (3) and (4) are applied, one has the following bracket expression for the stationarized autocorrelation function $R_{s}(\tau)$ of $S(t)$ : 


$$
\begin{aligned}
& R_{S}(\tau)=\lim _{N \rightarrow \infty} \frac{1}{(N+1) T} \sum_{m=0}^{N} \int_{m T}^{(m+1) T} E\{S(t) S(t+\tau)\} d t=
\end{aligned}
$$

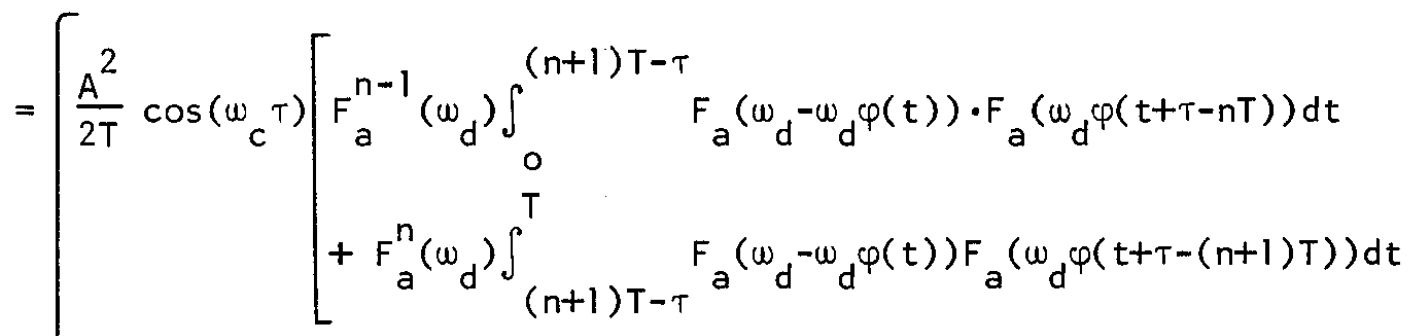

$$
\begin{aligned}
& \left\{\begin{aligned}
\frac{A^{2}}{2 T} \cos \left(\omega_{c} \tau\right)\left[\begin{array}{l}
n T \\
\int_{0}^{T-\tau} F_{a}\left(\omega_{d} \varphi(t+\tau)-\omega_{d} \varphi(t)\right) d t \\
+\int_{T-\tau}^{T} F_{a}\left(\omega_{d}-\omega_{d} \varphi(t)\right) F_{a}\left(\omega_{d} \varphi(t+\tau-T)\right) d t
\end{array}\right.
\end{aligned}\right. \\
& \lfloor\quad 0 \leq \tau \leq T
\end{aligned}
$$

where

$$
\begin{aligned}
& F_{a}(x)=E\left\{\exp \left(j \times a_{i}\right)\right\} \\
& \varphi(t)=\int_{0}^{t} g(\tau) d \tau
\end{aligned}
$$

It is obvious from expression (5) that the stationarized spectrum of $S(t)$ is band limited with band central frequencies the $\pm_{c}$. The low-pass autocorrelation $R_{L S}(\tau)$ in the binary case is then given by (7):

$$
\begin{aligned}
& R_{L S}(\tau)=\int \frac{A^{2}}{2 T} \int \cos ^{n-1} \omega_{d} \int_{0}^{(n+1) T-T} \cos \left(\omega_{d}-\omega_{d} \varphi(t)\right) \cos \left(\omega_{d} \varphi(t+\tau-n T)\right) d t \\
& +\cos ^{n} \omega_{d} \int_{(n+1) T-T}^{T} \cos \left(\omega_{d}-\omega_{d} \varphi(t)\right) \cos \left(\omega_{d} \varphi(t+T-(n+1) T)\right) d t \\
& n T \leq \tau \leq(n+1) T, n \geq 1 \\
& {\left[\begin{array}{l}
\frac{A^{2}}{2 T}\left[\int_{0}^{T-T} \cos \left(\omega_{d} \varphi(t+\tau)-\omega_{d} \varphi(t)\right) d t\right. \\
+\int_{T-\tau}^{T} \cos \left(\omega_{d}-\omega_{d} \varphi(t)\right) \cos \left(\omega_{d} \varphi(t+\tau-T)\right) d t
\end{array}\right.} \\
& 0 \leq \tau \leq \top
\end{aligned}
$$


There are a few signal-design observations that one can make right at this point. First of all, the angle $\omega_{\mathrm{d}}$ must be somewhere in the interval $(0, \pi)$. If $\omega_{\mathrm{d}}$ is equal to $\pi / 2$ (orthogonal signals), the autocorrelation is obviously zero for $\tau>2 T$ and the process $S(t)$ cannot be strictly frequency limited, then. If $\omega_{\mathrm{d}}$ is different from $\pi / 2$, the autocorrelation has the form of a decaying oscillation of period either $T$ if $\omega_{\mathrm{d}}$ lies in the interval $\left(0, \pi / 2\right.$, or $2 \mathrm{~T}$ if $\omega_{\mathrm{d}}$ lies in $(\pi / 2, \pi)$. Hence, for given pulse (given $\varphi(t))$ one expects sharper spectra (less modulated signal distortion) when the modulating signals $+\omega_{\mathrm{d}}$ and $-\omega_{\mathrm{d}}$ are not orthogonal.

Let us now call

$$
\begin{aligned}
f_{1}(\tau)= & \frac{A^{2}}{2 T} \int_{0}^{T-\tau} \cos \left(\omega_{d} \varphi(t+\tau)-\omega_{d} \varphi(t)\right) d t \\
& +\frac{A^{2}}{2 T} \int_{T-\tau}^{T} \cos \left(\omega_{d}-\omega_{d} \varphi(t)\right) \cos \left(\omega_{d} \varphi(t+\tau-T)\right) d t \\
f_{2}(\tau)= & \frac{A^{2}}{2 T} \int_{0}^{T-\tau} \cos \left(\omega_{d}-\omega_{d} \varphi(t)\right) \cos \left(\omega_{d} \varphi(t+\tau)\right) d t \\
& +\frac{A^{2}}{2 T} \cos \omega_{d} \int_{T-t}^{T} \cos \left(\omega_{d}-\omega_{d} \varphi(t)\right) \cos \left(\omega_{d} \varphi(t+T-T)\right) d t
\end{aligned}
$$

Then, application of the two last expressions to expression (7), gives the following expression for the low-pass waveform spectrum in the binary case:

$$
\begin{aligned}
F_{L S}(\omega)= & 2 \int_{0}^{T} f_{1}(\tau) \cos \omega \tau d \tau+ \\
& +2\left[\sum_{n=1}^{\infty} \cos ^{n-1}\left(\omega_{d}\right) \cos (n \omega T)\right] \int_{0}^{T} f_{2}(\tau) \cos (\omega \tau) d \tau \\
& -2\left[\sum_{n=1}^{\infty} \cos ^{n-1}\left(\omega_{d}\right) \sin (n \omega T)\right] \int_{0}^{T} f_{2}(\tau) \sin (\omega \tau) d \tau
\end{aligned}
$$

Introducing the functions

$$
k_{1}(\tau)=\frac{A^{2}}{4 T} \int_{0}^{T-\tau} \sin \left(\omega_{d} \varphi(t+\tau)+\omega_{d} \varphi(t)\right) d t
$$




$$
\begin{aligned}
& K_{2}(\tau)=\frac{A^{2}}{4 T} \int_{0}^{T-\tau} \sin \left(\omega_{d} \varphi(t+\tau)-\omega_{d} \varphi(t)\right) d t \\
& K_{3}(\tau)=\frac{A^{2}}{4 T} \int_{0}^{T-\tau} \cos \left(\omega_{d} \varphi(t+\tau)+\omega_{d} \varphi(t)\right) d t \\
& K_{4}(\tau)=\frac{A^{2}}{4 T} \int_{0}^{T-\tau} \cos \left(\omega_{d} \varphi(t+\tau)-\omega_{d} \varphi(t)\right) d t
\end{aligned}
$$

one finds from (10)

$$
\begin{aligned}
& F_{S L}(\omega)=\frac{2}{1+\cos ^{2} \omega_{d}-2 \cos \omega_{d} \cos \omega T}\left\{\int_{0}^{T} d \tau \cos \omega \tau\right. \text {. } \\
& {\left[\sin \omega_{d}\left[2 \cos \omega T+\cos ^{2} \omega_{d} \cos \omega T-\cos \omega_{d}\right] K_{1}(\tau)\right.} \\
& -\sin \omega_{d} \cos \omega T\left[\sin ^{2} \omega_{d}-2 \cos \omega_{d} \cos \omega T\right] k_{2}(\tau) \\
& +\cos \omega_{d}\left[2 \cos \omega T+\cos ^{2} \omega_{d} \cos \omega T-\cos \omega_{d}\right] K_{3}(\tau) \\
& \left.+\left[2+\cos ^{2} \omega_{d}-2 \cos \omega_{d} \cos \omega T+\cos ^{3} \omega_{d} \cos \omega T\right] k_{4}(\tau)\right] \\
& +\int_{0}^{T} d \tau \sin \omega \tau\left[\sin \omega_{d} \cos ^{2} \omega_{d} \sin \omega T k_{l}(\tau)\right. \\
& +\sin \omega_{d} \sin \omega T\left[2+\cos ^{2} \omega_{d}\right] k_{2}(\tau) \\
& +\cos ^{3} \omega_{d} \sin \omega T k_{3}(\tau) \\
& \left.\left.+\cos ^{3} \omega_{d} \sin \omega T k_{4}(\tau)\right]\right\}
\end{aligned}
$$

Expression (15) is a general formula for the low-pass spectrum of the modulated waveform $\mathrm{S}(\mathrm{t})$, when the series of modulating digits $\mathrm{a}_{\mathrm{i}}$ is a series of independent plus and minus ones. The pulse shape $g(t)$ is included in the functions $K_{1}(\tau), K_{2}(\tau), K_{3}(\tau)$, and $K_{4}(\tau)$ and the signaling frequency (frequency of transmission) is $\omega_{\mathrm{d}}$. One can work on (15) to find $\mathrm{F}_{\mathrm{LS}}(\omega)$ under specific pulse shapes and signaling frequencies or to establish "nice" conditions on the pulses to achieve sharp spectrum. 
The Spectrum in the Presence of Orthogonal Pulses -- We will choose the pulse $g(t)$ here to be equal to $1 / T$ for $0 \leq t \leq T$. Then $g(t)=t / T$ and the spectrum $F_{S L}(\omega)$ can easily be found in this case. The result in the general case is a complicated expression of $\sin \omega_{d}$ and $\cos \omega_{\mathrm{d}}$, though. One can find simple expressions of $\mathrm{F}_{\mathrm{SL}}(\omega)$ for special values of the frequency of transmission $\omega_{\mathrm{d}}$.

Indeed, it can easily be found that for $\omega_{\mathrm{d}}=\pi / 2$ (orthogonal signals) the low-pass spectrum is given by the following expression:

$$
F_{S L}(\omega)=\frac{A^{2} \pi^{2}}{4 T^{3}} \frac{1+\cos 2 \omega T}{\left[(\pi / 2 T)^{2}-\omega^{2}\right]^{2}}
$$

From (16) it is obvious that the low-pass spectrum for orthogonal signals and rectangular signaling pulse is a decaying sinusoidal function with two peaks at $\omega= \pm \pi / 2 \mathrm{~T}$. This same spectrum decreases as fast as $\omega^{4}$.

Another Expression for $\mathrm{F}_{\mathrm{SL}}(\omega)$-- Let us define the following new functions:

$$
\begin{aligned}
\mathrm{g}_{1}(\tau) & =\mathrm{k}_{3}(\tau)+\mathrm{k}_{4}(\tau) \\
\mathrm{g}_{2}(\tau) & =\mathrm{k}_{4}(\tau)-\mathrm{k}_{3}(\tau) \\
\mathrm{g}_{3}(\tau) & =\mathrm{k}_{1}(\tau)-\mathrm{k}_{2}(\tau) \\
\mathrm{g}_{4}(\tau) & =\cos \omega_{\mathrm{d}} \mathrm{k}_{4}(\mathrm{~T}-\tau)+\sin \omega_{\mathrm{d}} \mathrm{k}_{2}(\mathrm{~T}-\tau)+\cos \omega_{\mathrm{d}} \mathrm{k}_{3}(\mathrm{~T}-\tau)+ \\
& +\sin \omega_{\mathrm{d}} \mathrm{k}_{1}(\mathrm{~T}-\tau)
\end{aligned}
$$

Then, if one uses the above expressions to find $\mathrm{F}_{\mathrm{SL}}(\omega)$ one gets

$$
\begin{aligned}
F_{S L}(\omega) & =\int_{0}^{T}\left[g_{1}(\tau)+g_{4}(\tau)+g_{2}(\tau)\right] \cos \omega \tau d \omega \\
+ & \frac{2 \cos \omega T}{1+\cos ^{2} \omega_{d}-2 \cos \omega_{d} \cos \omega T} \int_{0}^{T}\left[\cos \omega_{d}\left(g_{1}(\tau)+g_{4}(\tau)\right)+\sin \omega_{d} g_{3}(\tau)\right] \cos \omega \tau d \tau \\
& -\frac{2 \sin \omega T}{1+\cos ^{2} \omega_{d}-2 \cos \omega_{d} \cos \omega T} \int_{0}^{T}\left[\cos \omega_{d}\left(g_{1}(\tau)+g_{4}(\tau)\right)+\sin \omega_{d} g_{3}(\tau)\right] \sin \omega \tau d \tau
\end{aligned}
$$


If we now assume that the pulse $\mathrm{g}(\mathrm{t})$ is smooth (all derivatives existing), so are $\mathrm{k}_{1}(\tau), \mathrm{k}_{2}(\tau)$, $\mathrm{k}_{3}(\tau), \mathrm{k}_{4}(\tau)$ and $\mathrm{g}_{1}(\tau), \mathrm{g}_{2}(\tau), \mathrm{g}_{3}(\tau), \mathrm{g}_{4},(\tau)$. $\mathrm{S}$, let us define the following Taylor expansions:

$$
\begin{aligned}
& g_{1}(\tau)+g_{4}(\tau)=\sum_{i=0}^{\infty} c_{i} \tau^{i} \\
& g_{2}(\tau)=\sum_{i=0}^{\infty} d_{i} \tau^{i} \\
& g_{3}(\tau)=\sum_{i=0}^{\infty} e_{i} \tau^{i}
\end{aligned}
$$

where

$$
\begin{aligned}
& c_{i}=\left[g_{1}^{(i)}(0)+g_{4}^{(i)}(0)\right] \frac{1}{i !} \\
& d_{i}=\frac{1}{i !} g_{2}^{(i)}(0) \\
& e_{i}=\frac{1}{i !} g_{3}^{(i)}(0)
\end{aligned}
$$

If we assume that the summation of the infinite Taylor series converges to the Fourier characteristics of the functions, one will get, by application of (22-27) to (21), an expression for $F_{S L}(\omega)$ which will be simple in the case of orthogonal signals $\left(\omega_{d}=\pi / 2\right)$. Indeed, we have then

$$
\begin{aligned}
F_{S L}(\omega)= & 2 \sin 2 \omega T \cdot \sum_{j=0}^{\infty} \frac{(-1)^{j}}{2 j+1}\left[k_{1}^{(2 j)}(T)-k_{2}^{(2 j)}(T)\right] \\
& +2 \cos 2 \omega T \cdot \sum_{j=0}^{\infty} \frac{(-1)^{j}}{2 j+2}\left[k_{1}^{(2 j+1)}(T)-k_{2}^{(2 j+1)}(T)\right] \\
& +2 \sum_{j=0}^{\infty} \frac{(-1)^{j}}{2 j+2}\left[k_{2}^{(2 j+1)}(T)+k_{1}^{(2 j+1)}(T)-k_{3}^{(2 j+1)}(0)-k_{4}^{(2 j+1)}(0)\right] \\
& +2 \cos \omega T \sum_{j=0}^{\infty} \frac{(-1)^{j}}{2 j+2}\left[k_{3}^{(2 j+1)}(T)+k_{4}^{(2 j+1)}(T)-2 k_{1}^{(2 j+1)}(0)-2 k_{2}^{(2 j+1)}(0)\right] \\
& +2 \sin \omega T \sum_{j=0}^{\infty} \frac{(-1)^{j}}{2 j+1}\left[2 k_{4}^{(2 j)}(T)+k_{2}^{(2 j)}(T)-k_{1}^{(2 j)}(T)+k_{1}^{(2 j)}(0)+k_{2}^{(2 j)}(0)\right]
\end{aligned}
$$


Based on expression (28), one can make some observations. From (11) and (12), one can easily find that for orthogonal signals $\left(\omega_{\mathrm{d}}=\pi / 2\right)$ one has

$$
k_{1}^{(3)}(T)-k_{2}^{(3)}(T)=-\frac{A^{2} \omega_{d}^{2}}{2 T} \dot{\varphi}(0)[\dot{\varphi}(0)+\dot{\varphi}(T)]
$$

We will remind the reader here that by definition

$$
\dot{\varphi}(t)=g(t) \leftarrow \text { the pulse, which is always nonnegative hence } \dot{\varphi}(0) \text { and } \dot{\varphi}(T)
$$

are always nonnegative and $k_{1}^{(3)}(T)-k_{2}^{(3)}(T)$ can be zero only if $\dot{\varphi}(0)$ and $\dot{\varphi}(T)$ are zero. On the other hand, for $\omega_{d}=\pi / 2$ still, $k_{1}^{(2)}(T)-k_{z}^{(2)}(T)=0$ for every pulse.

Conclusion -- Assuming that all the partial sums mentioned above converge and, for binary orthogonal signal case, one has a sharper low-pass spectrum $\mathrm{F}_{\mathrm{SL}}(\omega)$ than the one accomplished by the rectangular pulse (one of the sinusoidal terms decaying faster than $\omega^{-4}$ which is the order of decrease for the rectangular pulses (16)) if one chooses instead of a smooth signaling pulse that is zero at the beginning and the end of the signaling interval of length $\mathrm{T}$

\section{References}

1. R.R. Anderson and J. Salz, "Spectra of digital FM," B.S.T.J. $\underline{6}$ : July-August 1965.

2. W.R. Bennett and S.O. Rice, "Spectral density and autocorrelation functions associated with binary frequency shift keying," B.S.T.S. 42: Sept. 1963.

3. J. Sa1z, "Spectral density function of multilevel continuous phase FM," IEEE Trans. Inf. Th.: July 1965.

4. D. Gabor, "Theory of communication,” J. Inst. Elec. Engrs. 93 III: 429.

5. M.J. Lighthill, An Introduction to Fourier Analysis and Generalized Functions, Cambridge University Press, 1959.

6. P. Papantoni-Kazakos and I. Paz, "The effect of intersymbol interference on the performance of FM digital systems," Rice University, Electrical Engineering Department Report \#7403, 1974. 